\title{
Statistical analysis of markovian and related time-series data
}

\author{
Than Toe
}

The Markov chain model provides a useful representation of serial dependence in time-series data. It often happens that while a Markov model is applicable to an ideal series of observations what one observes in practice is a related series which is no longer markovian. For instance, one may observe a grouped chain defined on a new state-space which is a partition of the original state-space. Also, an observed chain might be error-contaminated. Moreover, the available data may be in an aggregate form. Practical circumstances such as these provided a motivation for a study in this thesis of estimation procedures based on certain functions of Markov processes.

Least-square method is adopted to obtain estimators of transition probabilities from aggregate data. Minimum chi-square estimators are obtained for parameters of grouped Markov chains; consistency and asymptotic normality of these estimators are established. Various questions related to grouping, such as loss of information are discussed and illustrated with some simulation studies. Other topics discussed include state-estimation and parameter estimation for autoregressive series contaminated by random noise.

Received 10 May 1977. Thesis submitted to La Trobe University, October 1976. Degree approved, May 1977. Supervisor: Dr I.V. Basawa. 